\title{
A DYNAMIC REGULATING MECHANISM FOR INCREASED AIRFLOW SPEED RANGE IN MICRO PIEZOELECTRIC TURBINES \\ Hailing Fu' ${ }^{l}$, Mario D'Auria ${ }^{1}$, Guangbin Dou ${ }^{l}$ and Eric M. Yeatman ${ }^{l}$ \\ ${ }^{1}$ Departmen of Electrical and Electronic Engineering, Imperial College London, London, UK
}

\begin{abstract}
The paper reports the design and fabrication of a micro-planar spring for a dynamic regulating mechanism to decrease the cut-in (start-up) airflow speed of a piezoelectric turbine. This mechanism is implemented by adjusting the magnetic coupling between the turbine rotor and a piezoelectric cantilever using the spring. Varied spring shapes and dimensions were analyzed with the finite element method (FEM) to optimize the structure. A micro spring with an ultra-low spring constant of $0.78 \mathrm{~N} / \mathrm{m}$ was fabricated from titanium foil by laser machining. The spring was installed into a miniaturized air turbine to achieve the self-regulation. The cut-in speed was $2.34 \mathrm{~m} / \mathrm{s}$, showing a 30\% improvement against a non-regulated turbine.
\end{abstract}

\section{INTRODUCTION}

In recent years, wireless sensor networks have been adopted extensively to collect useful data in a range of applications, including smart agriculture, the internet of vehicles and machine health monitoring [1-3]. However, sensors in the networks are generally powered by batteries which need to be replaced or recharged regularly [4]. Energy harvesting from airflow is a promising alternative to make sensor nodes autonomous. Miniaturization of conventional turbines is a feasible approach for airflow energy harvesting research. Work has been done in several groups based on this principle. Bansal et al. [5] developed a centimeter-scale electromagnetic air turbine for duct sensing applications. The device achieved an output power of $4.3 \mathrm{~mW}$ at airflow speed $10 \mathrm{~m} / \mathrm{s}$ and operated at flow speeds down to $4.5 \mathrm{~m} / \mathrm{s}$. Myers et al. [6] demonstrated a piezoelectric windmill in which a set of 18 piezoelectric bimorphs was installed and plucked by three fan rotors $(\varnothing 127 \mathrm{~mm})$ via a crank mechanism. The device provided $5 \mathrm{~mW}$ continuous power at $4.47 \mathrm{~m} / \mathrm{s}$ airspeed and the threshold speed was $2.41 \mathrm{~m} / \mathrm{s}$. Fu et al. [7] adapted the magnetic plucking method into a piezoelectric turbine, allowing the piezoelectric beam to operate without impact. A peak output power of $159 \mu \mathrm{W}$ was obtained at $2.7 \mathrm{~m} / \mathrm{s}$.

However, these devices generally work at high air speeds or have a relatively low output power when the operating airspeed range is extended. In order to increase the operating range and to maintain the output power, a dynamic regulating mechanism for piezoelectric turbines is designed in this paper.

\section{PIEZOELECTRIC TURBINE WITH SELF- REGULATION}

The schematic of the piezoelectric turbine and the self-regulating mechanism is illustrated in Figure 1. The turbine has six inlets arranged on the lateral sides of the hexagonal prism casing, allowing the device to operate with airflow from any direction. The turbine's transduction is achieved by non-contact plucking of a piezoelectric cantilever by the magnetic force which is formed by magnets on the turbine rotor and the cantilever free end. The cantilever mounted on the turbine casing is plucked once per motion cycle and operates in a free vibration form after each plucking.

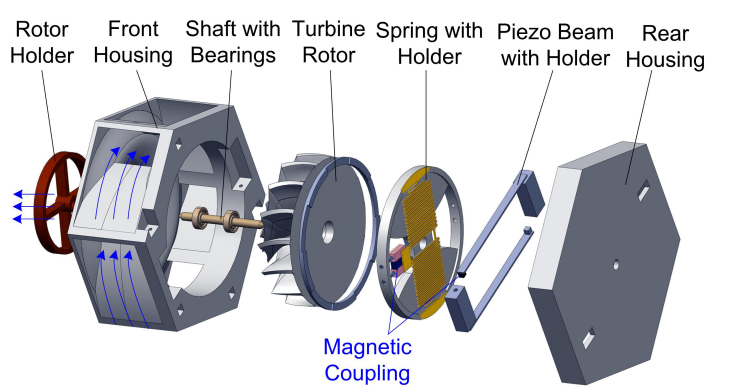

Figure 1: Schematic of the miniaturized piezoelectric wind turbine with a dynamic regulating mechanism.

The main resistance impeding the turbine to start up is the magnetic force imposed on the turbine rotor. Once the turbine rotates, the driving torque and the inertia of the turbine rotor can easily conquer the magnetic resistance. In order to decrease the strength of the magnetic coupling in the static situation and intensify the strength immediately after the turbine starts up to enhance the power output, a self-regulating mechanism was designed to dynamically adjust the relative radial gap between the magnets in response to airflow speed.

The main components to realize the mechanism are a micro-spring and two guide rails. A magnet is mounted on the guide rails with two sliders which can only move along the rails. This ensures the magnet does not have relative tangential movement to the turbine rotor. The spring is rigidly connected with the magnet as shown in Figure 1. The length of the spring is passively controlled by the centrifugal force of the rotating magnet with respect to different rotational speeds. Hence, the gap between the magnets is passively adjusted in response to airflow speed.

The size of the rotating magnet is $5 \mathrm{~mm} \times 1.5 \mathrm{~mm} \times$ $1 \mathrm{~mm}$. The diameter of the turbine rotor is $\varnothing 27 \mathrm{~mm}$. The initial radial position of the rotating magnet is $8 \mathrm{~mm}$ from the rotation axis. The centrifugal force generated by rotation is calculated as shown in Figure 2.

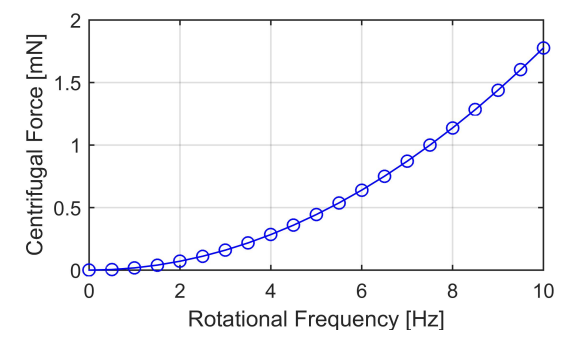

Figure 2: Calculated centrifugal force generated by the magnet at the initial radial location versus frequency. 
The force is on millinewton scale, which means the spring should be soft enough to intensify the magnetic coupling quickly after start up. The length of the stretched spring at a certain frequency, $\omega$, can be given as

$$
l_{s p}=\frac{k_{s} l_{0}}{k_{s}-m_{r m} \omega^{2}},
$$

where $k_{s}$ is the spring constant, $l_{0}$ is the free length of the spring and $m_{r m}$ is the mass of the rotating magnet.

The relationship between the lengths of spring versus rotational frequency for different spring constants are illustrated in Figure 3. The maximum deformation of the spring is determined by a mechanical stopper on the turbine rotor. In order to implement the passive regulating mechanism, the spring constant should be on the "N/m" scale and as low as possible, but the elastic limit and the stiffness of the spring to restore its original shape under a static condition should be considered.

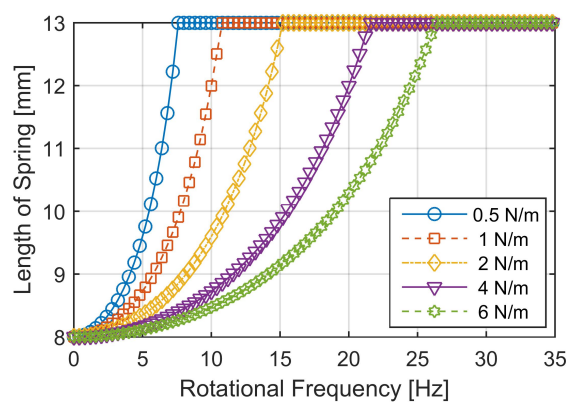

Figure 3: Simulated spring length versus rotational frequency for different spring constants.

\section{MICRO-PLANAR SPRING}

In order to design a spring with a low spring constant, different shapes of springs are first investigated using FEM. Four types of springs with uniform dimensions are simulated, including square-shape, v-shape, sine-shape and $\mathrm{u}$-shape. As illustrated in Table 1, the u-shape spring has the lowest spring constant among the four types, but further consideration is needed to decrease the spring constant.

Table 1: Simulated spring constant of springs with different shapes and uniform dimension.

\begin{tabular}{|c|c|c|}
\hline \multicolumn{2}{|c|}{ Shape } & $\begin{array}{c}\text { Spring constant } \\
(\mathrm{N} / \mathrm{mm})\end{array}$ \\
\hline Square shape & क्षापाला & 0.590 \\
\hline V-shape & MWWW & 0.580 \\
\hline Sine shape & IMWW & 0.583 \\
\hline U-shape & ษกกกกกก" & 0.311 \\
\hline
\end{tabular}

Different structural parameters of the u-shape spring are considered, including spring turns, the length and width of each turn and the width of the spring beam. The model of the u-shape spring is shown in Figure 4. Considering the limited space on the turbine rotor, the operating direction of the spring is designed to be perpendicular to its length direction. The simulated results are illustrated in Figure 5. The number of turns has a significant impact on the constant, decreasing the spring constant with increasing turns. The optimized spring has the spring constant of
$1.58 \mathrm{~N} / \mathrm{m}$, as shown in Figure 5. The width of the spring beam is $100 \mu \mathrm{m}$ and the width and length of each turn are $10 \mathrm{~mm}$ and $0.7 \mathrm{~mm}$ respectively.

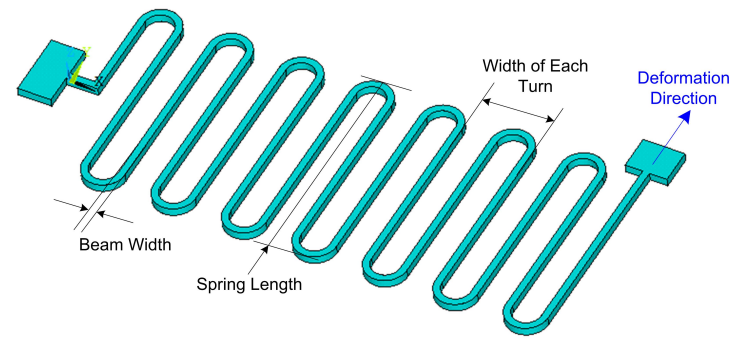

Figure 4: U-shape spring model with its structural parameters and operating orientation.
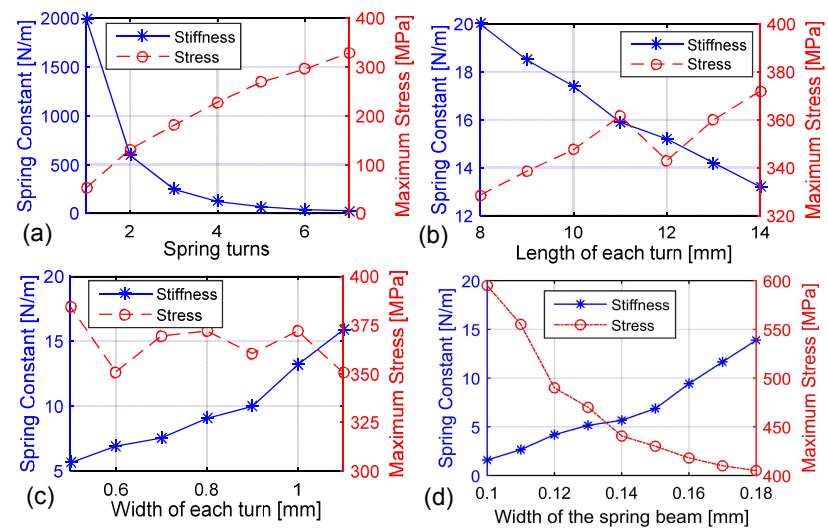

Figure 5: Simulated spring constant and maximum stress of the u-shape spring against different parameters. (a) Spring turns, (b) length of each turn, (c) width of each turn and $(d)$ width of the spring beam.

\section{FABRICATION AND TESTING}

For micro-spring manufacturing, the general method is silicon-based fabrication [8]. However, the material that can be used for this method is limited and the spring generally cannot produce a large distortion [9]. Laser machining, as an alternative solution, is capable of cutting materials in a wide range [10]. To achieve the ultra-low spring constant and large distortion, titanium foil is chosen for its advantage in flexibility and high elastic limit (786$910 \mathrm{MPa}[11])$.
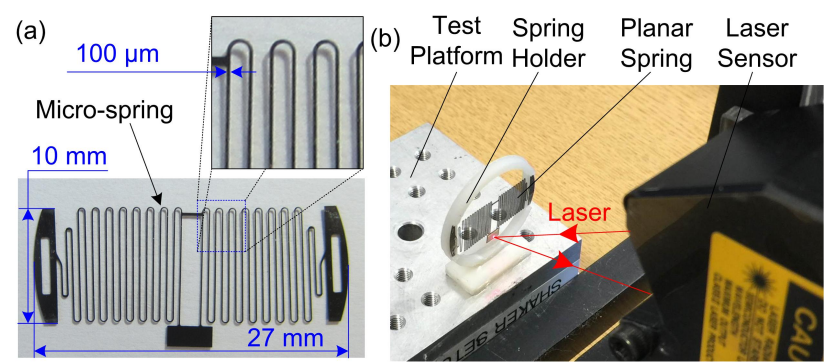

Figure 6: (a) Prototype of the micro-planar spring and (b) experimental set-up for the spring constant measurement.

The fabricated spring is shown in Figure 6(a). In order to measure the spring constant, an experimental set-up was built as shown in Figure 6(b). The spring is fixed on a spring holder which is rigidly mounted on a testing bench. 
A laser sensor is used to measure the displacement of the spring caused by the vertical vibration.

The constant was measured based on the impulse excitation technique [12]. A vertical impulse excitation was imposed on the testing bench close to the spring. The vibration data of the spring were collected from the sensor. The results are shown in Figure 7. The vertical vibration mode of the spring is excited by the impulse input and its resonant frequency in the vertical direction is $7.62 \mathrm{~Hz}$. The spring constant can be calculated using

$$
k_{s}=m \omega_{n}^{2} \text {, }
$$

where $m$ is the mass of the spring and $\omega_{n}$ is the resonant frequency. The spring constant is $0.78 \mathrm{~N} / \mathrm{m}$ based on the above equation.
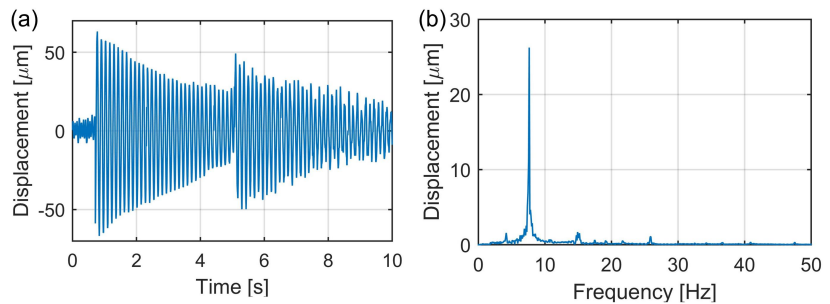

Figure 7: Vibration of the spring, showing its resonant frequency in vertical orientation.

\section{RESULTS}

In order to examine the effect of the self-regulation, a simplified equivalent experimental set-up was built, as illustrated in Figure 8. A DC-motor is employed as an analogy to the turbine rotor. The regulating mechanism is implemented on a rotating disk with the same diameter as the rotor. The plate is mounted on the motor's shaft rigidly. A piezoelectric beam is installed on an adjustable platform with micrometers controlling the position of the beam in 3 dimensions accurately. The vertical gap between magnets is $3 \mathrm{~mm}$ and the initial radial position of the sliding magnet is $5 \mathrm{~mm}$. The cantilever is connected with a $100 \mathrm{k} \Omega$ resistor where the output voltage is measured.

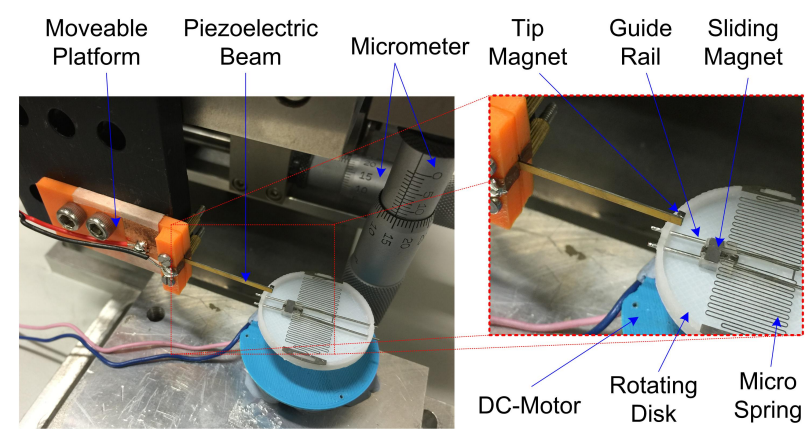

Figure 8: Equivalent experimental set-up to examine the self-regulating mechanism.

The prototype was tested against the rotational frequency of the motor. The output voltage at $11 \mathrm{~Hz}$ and $21 \mathrm{~Hz}$ is presented in Figure 9, showing the variation of the amplitude of the output voltage caused by the changing magnetic coupling. The peak output voltage is magnified 6.44 times from $0.41 \mathrm{~V}$ at $11 \mathrm{~Hz}$ to $2.64 \mathrm{~V}$ at $21 \mathrm{~Hz}$, which implies the intensification of the magnetic coupling with the stretch of the planar spring caused by the increased rotational frequency.
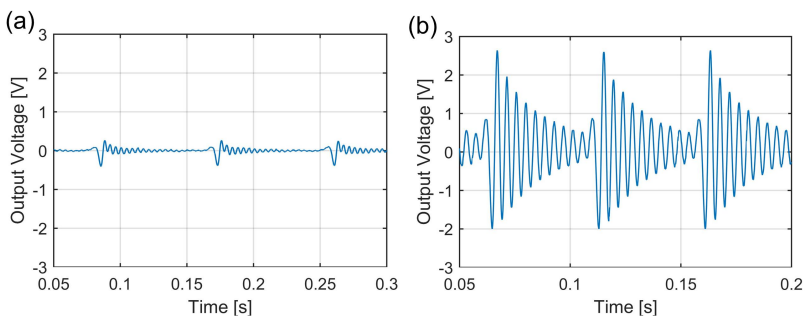

Figure 9: Output voltage of the piezoelectric beam at (a) $11 \mathrm{~Hz}$ and (b) $21 \mathrm{~Hz}$.

In order to visualize the deformation of the microspring, the fast VGA camera Pike F-032C (208 fps) was employed to record the regulating effect against the rotational frequency of the disk. The result is shown in Figure 10. The spring is unstretched initially and reaches its maximum displacement, $5 \mathrm{~mm}$, at $17.9 \mathrm{~Hz}$. We believe that the difference between the theoretical analysis in Figure 3 and the experimental results is caused by the fabrication inaccuracies and friction in the device.

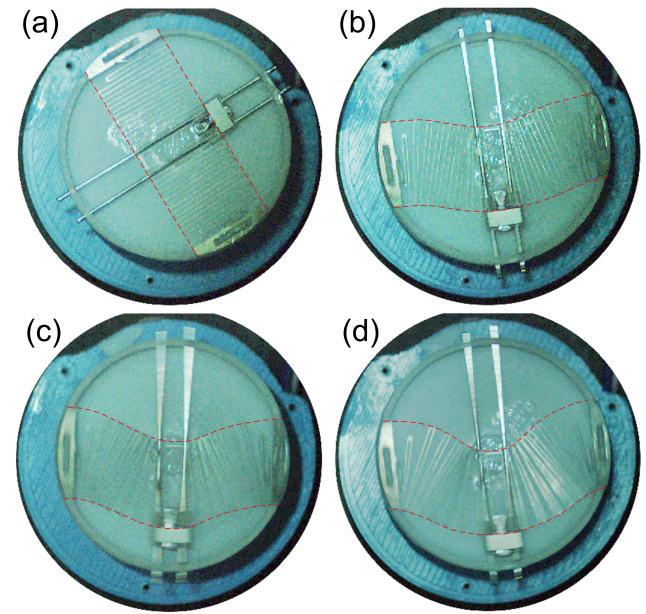

Figure 10: Deformation of the micro-spring for different frequencies with the envelope of the edges indicated. (a) Static, (b) $11.1 \mathrm{~Hz}$, (c) $14.2 \mathrm{~Hz}$ and (d) $17.9 \mathrm{~Hz}$.

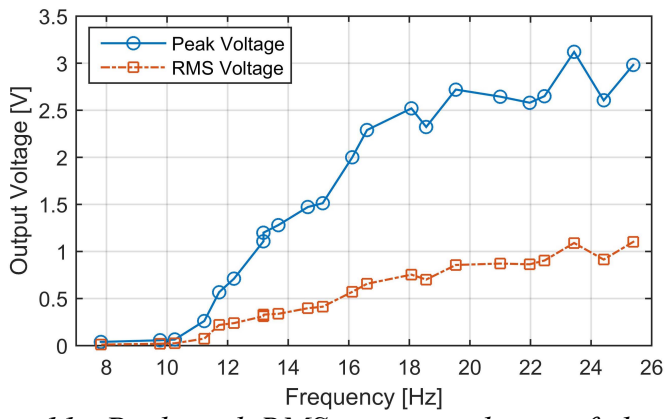

Figure 11: Peak and RMS output voltage of the beam against the rotational frequency of the rotating disk.

Figure 11 illustrates the peak and RMS output voltage against the rotational frequency of the rotor. The regulating behavior happens from $10 \mathrm{~Hz}$ to $18 \mathrm{~Hz}$. The output voltage at low frequency $(<10 \mathrm{~Hz})$ is ignorable, which means the energy converted by the piezoelectric beam is marginal. 
Hence, the only energy consumption at low frequency is caused by the friction in the system. The system, therefore, is much easier to start up. The output voltage is improved at high frequencies $(>18 \mathrm{~Hz})$, which ensures the output power of the device after start-up.

The regulating mechanism was then implemented in a piezoelectric turbine and tested in a wind tunnel as shown in Figure 12. The overall dimension of the turbine is $\varnothing 37$ $\mathrm{mm} \times 18 \mathrm{~mm}$. The device was installed in the test section of the wind tunnel. The airflow speed was measured by a pitot tube in parallel with the turbine.
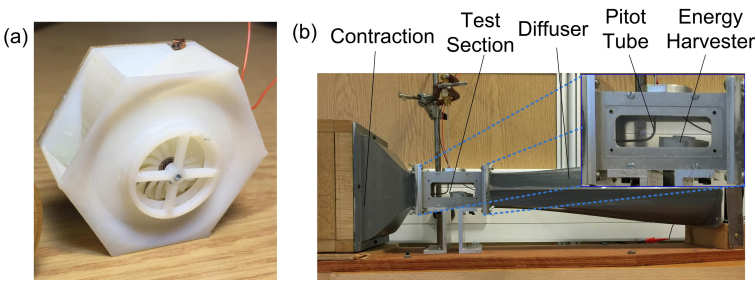

Figure 12: Prototype and experimental set-up. (a) Piezoelectric turbine and (b) miniature wind tunnel.

The turbine was tested at different airflow speeds. The output power and rotational speed of the turbine rotor is presented in Figure 13. The turbine started up at $2.34 \mathrm{~m} / \mathrm{s}$ and operated with airflow speed down to $1.87 \mathrm{~m} / \mathrm{s}$. A peak output power of $742 \mu \mathrm{W}$ was obtained at $4.2 \mathrm{~m} / \mathrm{s}$. A turbine without the regulating mechanism was also measured and the cut-in speed was $3.2 \mathrm{~m} / \mathrm{s}$. Therefore, the self-regulating mechanism has decreased the cut-in speed by $30 \%$.

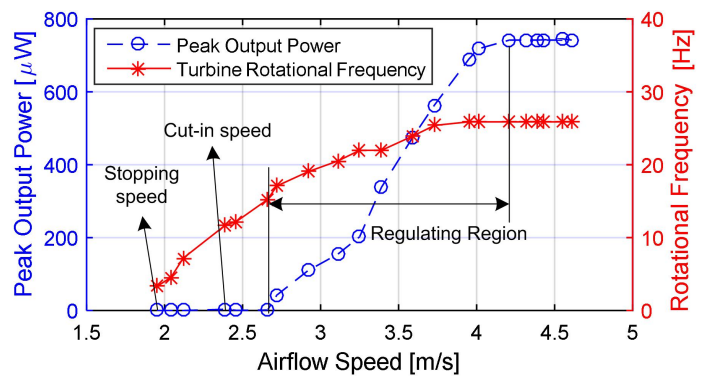

Figure 13: Peak output power and rotational frequency of the turbine rotor against airflow speed with a $100 \mathrm{k} \Omega \mathrm{load}$.

\section{CONCLUSIONS}

This paper presents a micro-spring-based dynamic regulating mechanism for increased airflow speed range in micro-piezoelectric turbines. This mechanism passively adjusts the magnetic coupling in the turbine using the spring, enabling the coupling to be weak at low airflow speeds and intensified at high airflow speeds. Hence the turbine has a low cut-in speed and the output power is ensured when it starts up.

A micro-planar spring with ultra-low spring constant, $0.78 \mathrm{~N} / \mathrm{m}$, was designed with the optimized shape and structural parameters. The spring was fabricated from titanium foil using laser cutting with a spring beam width of $100 \mu \mathrm{m}$. The spring was then installed onto a rotating plate on a DC motor to examine the effect of the regulating mechanism.

Eventually, the regulating mechanism was achieved in a piezoelectric turbine with the overall size of $\varnothing 37 \mathrm{~mm} \times 18$ $\mathrm{mm}$. The cut-in speed is $2.34 \mathrm{~m} / \mathrm{s}$, showing a $30 \%$ improvement against a non-regulated turbine. A peak output power of $742 \mu \mathrm{W}$ was measured at $4.21 \mathrm{~m} / \mathrm{s}$ with a $100 \mathrm{k} \Omega$ load.

\section{ACKNOWLEDGEMENTS}

The work is supported by the Department of Electrical and Electronic Engineering, Imperial College London and the China Scholarship Council (CSC). We would also like to thank Prof. Andrew Holmes and Mr. Tao Li for use of apparatus and valuable suggestions.

\section{REFERENCES}

[1] C.-T. Hsu, G.-Y. Huang, H.-S. Chu, B. Yu, and D.-J. Yao, "Experiments and simulations on low-temperature waste heat harvesting system by thermoelectric power generators", Applied Energy, vol. 88, pp. 1291-1297, 2011.

[2] C. Shen, X. Wang, W. Zhang, and F. Kang, "A high-performance three-dimensional micro supercapacitor based on self-supporting composite materials", Journal of Power Sources, vol. 196, pp. 10465-10471, 2011.

[3] P. D. Mitcheson, E. M. Yeatman, G. K. Rao, A. S. Holmes, and T. C. Green, "Energy harvesting from human and machine motion for wireless electronic devices", Proceedings Of the IEEE, vol. 96, pp. 1457-1486, 2008.

[4] E. M. Yeatman, "Energy harvesting from motion using rotating and gyroscopic proof masses", Proceedings of the Institution of Mechanical Engineers, Part C: Journal of Mechanical Engineering Science, vol. 222, pp. 27-36, 2008.

[5] A. Bansal, D. A. Howey, and A. S. Holmes, "CM-scale air turbine and generator for energy harvesting from low-speed flows", in Solid-State Sensors, Actuators and Microsystems Conference. TRANSDUCERS 2009, pp. 529-532.

[6] R. Myers, M. Vickers, H. Kim, and S. Priya, "Small scale windmill", Applied Physics Letters, vol. 90, pp. 054106, 2007.

[7] H. Fu and E. M. Yeatman, "A miniature radial-flow wind turbine using piezoelectric transducers and magnetic excitation", Journal of Physics: Conference Series, 2015, in press.

[8] L. Sui, Z. Wang, G.-C. Shi, and G.-Z. Li, "MEMS variable stiffness spring and its application in fuze", Sensors \& Transducers, vol. 168, pp. 101-107, 2014.

[9] G. Li, L. Sui, and G. Shi, "Study on the Linearly Range of S-Shaped MEMS Planar Micro-spring", TELKOMNIKA Indonesian Journal of Electrical Engineering, vol. 10, pp. 1327-1332, 2012.

[10] G. Chryssolouris, Laser machining: theory and practice, Springer Science \& Business Media, 2013.

[11] www.azom.com/properties.aspx?articleid $=1547$

[12]W. G. Halvorsen and D. L. Brown, "Impulse technique for structural frequency response testing", Sound and Vibration, vol. 11, pp. 8-21, 1977.

\section{CONTACT}

*H. Fu, tel: +44 (0)7761 494934; h.fu14@imperial.ac.uk 